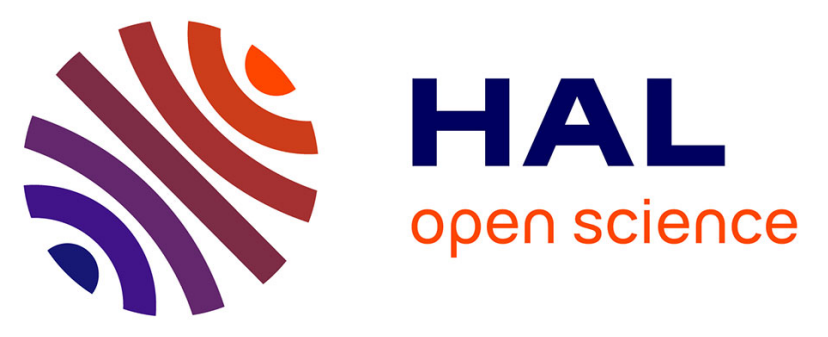

\title{
Plasma proprotein convertase subtilisin kexin type 9 is a heritable trait of familial combined hyperlipidemia
}

\author{
Martijn C.G.J. Brouwers, Marleen Mj van Greevenbroek, Jason Troutt, \\ Angela Bonner Freeman, Ake Lu, Nicolaas C Schaper, Robert John Konrad, \\ Coen D.A. Stehouwer
}

\section{To cite this version:}

Martijn C.G.J. Brouwers, Marleen Mj van Greevenbroek, Jason Troutt, Angela Bonner Freeman, Ake $\mathrm{Lu}$, et al.. Plasma proprotein convertase subtilisin kexin type 9 is a heritable trait of familial combined hyperlipidemia. Clinical Science, 2011, 121 (9), pp.397-403. 10.1042/CS20110129 . hal-00717078

\section{HAL Id: hal-00717078 \\ https://hal.science/hal-00717078}

Submitted on 12 Jul 2012

HAL is a multi-disciplinary open access archive for the deposit and dissemination of scientific research documents, whether they are published or not. The documents may come from teaching and research institutions in France or abroad, or from public or private research centers.
L'archive ouverte pluridisciplinaire HAL, est destinée au dépôt et à la diffusion de documents scientifiques de niveau recherche, publiés ou non, émanant des établissements d'enseignement et de recherche français ou étrangers, des laboratoires publics ou privés. 


\section{Plasma proprotein convertase subtilisin kexin type 9 is a heritable trait of familial combined hyperlipidemia}

Martijn C.G.J. Brouwers ${ }^{1,2,3}$, Marleen M.J. van Greevenbroek ${ }^{1,3}$, Jason S. Troutt ${ }^{4}$, Angela Bonner Freeman ${ }^{4}$, Ake Lu ${ }^{5}$, Nicolaas C. Schaper ${ }^{2}$, Robert J. Konrad ${ }^{4}$, Coen D.A. Stehouwer ${ }^{1,3}$

1,2,3 Department of Internal Medicine, Division of General Internal Medicine ${ }^{1}$ and Endocrinology ${ }^{2}$ and Laboratory of Metabolism and Vascular Medicine ${ }^{3}$, Cardiovascular Research Institute Maastricht, Maastricht University Medical Center, Maastricht, the Netherlands

4

Lilly Research Laboratories, Eli Lilly and Company, Indianapolis, USA

5 Departments of Human Genetics and Pediatrics, David Geffen School of Medicine, University of California Los Angeles, Los Angeles, California, USA

Abbreviated title: plasma PSCK9 in FCHL

Address all correspondence to:

Martijn C.G.J. Brouwers, MD, PhD

Department of Medicine and Endocrinology

PO Box 5800

6202 AZ Maastricht

the Netherlands

tel.: +31 (0)43-388 21 29, fax: +31 (0)43-3670916

martijn.brouwers@maastrichtuniversity.n1

Key words: dyslipidemia, cholesterol, statin, SREBP2, PCSK9 


\begin{abstract}
Objective: To study the relation between circulating proprotein convertase subtilisin kexin type 9 (PCSK9) and familial combined hyperlipidemia (FCHL) and - when positive - to determine the strength of its heritability.

Methods: Plasma PCSK9 levels were measured in FCHL patients $(n=45)$, normolipidemic relatives $(n=139)$ and their spouses $(n=72)$. In addition, eleven FCHL patients were treated with atorvastatin to study the response in PCSK9 levels.

Results: PCSK9 levels were higher in FCHL patients versus normolipidemic relatives and spouses: 96.1 versus 78.7 and $82.0 \mathrm{ng} / \mathrm{mL}, \mathrm{p}=0.004$ and $\mathrm{p}=0.002$, respectively. PCSK9 was significantly associated with both triglycerides and apolipoprotein B levels $(\mathrm{p}<0.001)$. The latter relation was accounted for by LDL-apoliprotein $\mathrm{B}(\mathrm{r}=0.31, \mathrm{p}=0.02)$, not by VLDL-apolipoprotein $B(r=0.09, p=0.49)$ in a subgroup of subjects $(n=59)$. Heritability calculations for PCSK9 using SOLAR and FCOR yielded estimates of 67$84 \%$, respectively $(\mathrm{p}<0.0001)$. PCSK9 increased from 122 to $150 \mathrm{ng} / \mathrm{mL}$ in eleven FCHL patients treated with atorvastatin $40 \mathrm{mg}$ once daily for 8 weeks $(\mathrm{p}=0.018)$.
\end{abstract}

Conclusions: Plasma PCSK9 is a heritable trait associated with both FCHL diagnostic hallmarks. These results combined with the significant rise in PCSK9 levels after statin therapy warrant further studies to unravel the exact role of PCSK9 in the pathogenesis and treatment of this highly prevalent genetic dyslipidemia. 


\section{Introduction}

Familial combined hyperlipidemia (FCHL) is the most prevalent genetic dyslipidemia in Western Society (estimated prevalence 1:100) and accounts for a substantial proportion of cases with premature myocardial infarction [1]. The current opinion about its pathogenesis is that the typical dyslipidemia, i.e. elevated plasma apolipoprotein B and triglycerides levels, is the consequence of hepatic VLDL overproduction, at a background of hepatic steatosis and insulin resistance, combined with an impaired clearance of remnants and LDL particles [2]. Several genetic defects have already been implicated in the clearance pathway, such as the apolipoprotein A1/C3/A4/A5 gene cluster and the lipoprotein lipase gene [3-5].

Proprotein convertase subtilisin kexin type 9 (PCSK9) is an important, inverse regulator of LDL particle clearance, since it promotes the degradation of the LDL receptor in hepatocytes by two different pathways, either intracellularly or as a systemically secreted protein that binds the LDL receptor resulting in their combined internalization and subsequent degradation [6]. A high expression of PCSK9 is therefore positively associated with plasma LDL cholesterol levels [6]. Of interest, recent studies have suggested that PCSK9 is also implicated in the regulation of VLDL production $[7,8]$. PCSK9 is therefore an intriguing candidate to evaluate its involvement in the FCHL phenotype.

The systemic release of PCSK9 enables its measurement in plasma, which can be used to gain more insight in relation of PCSK9 with lipoprotein metabolism in large cohort studies. In the present study, we have measured plasma PCSK9 in a cohort of FCHL patients, normolipidemic relatives and their spouses to examine its relation with the FCHL phenotype and - when positive - to determine the strength of its heritability. Furthermore, a subgroup of FCHL patients was treated with standard lipid lowering therapy to study the response in PCSK9 levels.

\section{Methods}

Subjects

FCHL patients, normolipidemic relatives and their spouses visited our lipid clinic in the period 2003-2005, after an overnight fast, three days abstinence from alcohol and two weeks withdrawal from lipid-lowering medication [9]. Diagnosis of FCHL affected state was based on plasma apolipoprotein B levels $>1.2 \mathrm{~g} / \mathrm{L}$ and plasma triglycerides levels $>$ $1.5 \mathrm{mmol} / \mathrm{L}$ [10]. Relatives of FCHL patients who did not fulfill to these criteria at the time of their visit were designated as 'normolipidemic relatives'. These subjects were included as an additional control group, since previous studies have shown that several metabolic and vascular disturbances, such as fatty liver and arterial stiffness, are not only confined to FCHL patients, but are also present in normolipidemic (NL) relatives [11, 12].

In addition, a subgroup of FCHL patients $(\mathrm{n}=11)$ also visited our clinic before and after eight weeks' treatment with atorvastatin $40 \mathrm{mg}$ once daily. This substudy was designed as 
a single arm, open-label study, without placebo controls. Subjects were asked to stop any lipid lowering medication 4 weeks prior to initiation of the study ( 5 subjects had been treated with statin monotherapy, 3 subjects with a combination therapy of a statin and fibrate, and the remaining 3 subjects had not received any lipid lowering therapy at the start of the study). Atorvastatin 40mg at bedtime was prescribed for 8 weeks as monotherapy.

All subjects gave written informed consent. This study was approved by the local medical ethical review board of the Maastricht University.

\section{Measurements}

Subjects were weighed in their underwear. BMI was calculated as weight divided by height squared. Waist circumference was measured in the standing position midway between the lower rib and the spina iliaca anterior superior.

Systolic and diastolic blood pressures were measured twice in sitting position after 10 min of rest (Omron 705CP; OMRON Healthcare GmbH, Hamburg, Germany). Blood was collected after an overnight fast in pre-cooled EDTA tubes. After centrifugation at $3000 \mathrm{rpm}$ for $15 \mathrm{~min}$ at $4^{\circ} \mathrm{C}$, plasma aliquots were stored at $-80^{\circ} \mathrm{C}$. Plasma triglycerides, apolipoprotein $\mathrm{B}$, total cholesterol, HDL cholesterol, insulin and glucose were measured as described previously [9]. An updated computer model, which was based on formulas introduced by Matthews et al. in 1985 [13], was used to estimate insulin resistance (HOMA-IR) (http://www.dtu.ox.ac.uk).

Plasma PCSK9 levels were measured in stored samples with the previously described PCSK9 dual monoclonal antibody sandwich ELISA [14]. Samples were shipped on dry ice and stored at $-70^{\circ} \mathrm{C}$ prior to analysis. The freeze-thaw stability was excellent with $>90 \%$ recovery even after 4 freeze-thaw cycles. The intra assay coefficient of variation $(\mathrm{CV} \%)$ was $3.9-8.9 \%$ [15]. The recently reported diurnal variation in plasma PCSK9 levels has introduced some additional scattering in PCSK9 concentrations [16], since blood was drawn between 9:00 AM and 12:00 noon, irrespective of the affected state. In a subset of subjects, apolipoprotein B levels were also determined in the VLDL and LDL subfractions, isolated by ultracentrifugation as described by Redgrave et al [17]. Apolipoprotein B levels in the VLDL subfraction were measured by gel electrophoresis according to the method of Karpe and Hamsten [18], whereas apolipoprotein B levels in the LDL subfraction were measured using the same method as in total plasma.

\section{Statistical analyses}

Data are presented as mean \pm SD or as median (interquartile range) in case of non-normal distribution. Non-normally distributed parameters (triglycerides, HOMA-IR and PCSK9) were $\log$ transformed prior to analyses. First, differences in PCSK9 concentrations between FCHL patients, NL relatives and their spouses were calculated with linear regression with adjustment for age and sex. Next, the associations between PCSK9 (main determinant) and apolipoprotein B and triglycerides (main outcomes) were determined. These analyses were adjusted for age, sex and family state, i.e. FCHL patient (yes or no) or NL relative (yes or no), included as dummy variables.

The change in plasma PCSK9 levels after treatment with atorvastatin was analyzed with the paired-samples T-test. 
All statistical analyses were carried out with the use of the Statistical Package of Social Sciences (SPSS) version 16.0 for Windows (SPSS, Inc., Chicago, Illinois, USA).

\section{Heritability calculations}

Heritability calculations were conducted to estimate to what extent the variation in plasma PCSK9 levels could be accounted for by genetic factors. Heritability calculations were done exactly as described before [9]. In short, the FCOR subprogram of the SAGE software package [19] was used to estimate the intraclass correlations for sibling pairs. The maximum heritability estimate $\left(\mathrm{h}^{2}\right)$ can be derived from the intraclass correlation $(\mathrm{r})$ : $\mathrm{h}^{2}=2 \mathrm{r}$. In addition, the variance component model implemented in SOLAR [20], which includes all family relationships, was also used to estimate the familiality of PCSK9. Heritability estimates were also calculated for the apolipoprotein B and normalized triglycerides traits, both used as a reference. The transformation to normality was made prior to the heritability calculations.

\section{Results}

General characteristics of study population

Characteristics of FCHL patients $(\mathrm{n}=45)$, normolipidemic $(\mathrm{NL})$ relatives $(\mathrm{n}=139)$ and their spouses $(\mathrm{n}=72)$ are displayed in Table 1 . NL relatives were significantly younger and there were more women in the FCHL group $(p=0.08)$ when compared to their spouses. Both FCHL patients and NL relatives were more abdominally obese and exhibited higher systolic blood pressure, plasma triglycerides and HOMA-IR levels when compared to their spouses. Plasma apolipoprotein B and diastolic blood pressure were elevated in FCHL patients when compared to their NL relatives and spouses (Table 1).

Plasma PCSK9 levels in FCHL patients, $N$ L relatives and their spouses

Plasma PCSK9 levels (interquartile range) were significantly higher in FCHL patients in comparison to their spouses: $96.1 \mathrm{ng} / \mathrm{mL}(73.7-132.9)$ versus $82.0 \mathrm{ng} / \mathrm{mL}(65.3-97.9)$, $\mathrm{p}=0.002$, and also to their NL relatives: $96.1 \mathrm{ng} / \mathrm{mL}(73.7-132.9)$ versus $78.7 \mathrm{ng} / \mathrm{mL}$ $(60.6-100.3), p=0.004$. No differences were observed between NL relatives and spouses $(\mathrm{p}=0.51)$.

Additional adjustment for history of use of lipid-lowering and antihypertensive medication, BMI or waist circumference, HOMA-IR, current smoking and systolic/diastolic blood pressure did not affect the outcomes substantially (data not shown). Furthermore, re-analysis with only FCHL patients naïve to any lipid lowering medication $(n=21)$ also demonstrated significantly higher PCSK9 levels when compared to spouses $(p=0.04)$, indicating that statin therapy did not account for the observed differences in PCSK9 levels between the groups of interest.

Relation of plasma PCSK9 levels with diagnostic hallmarks of FCHL

To gain more insight in to which diagnostic hallmarks PCSK9 levels are related, we subsequently constructed multivariate regression models with plasma apolipoprotein B and triglycerides levels as dependent variables. Age- and sex-adjusted regression slopes for PCSK9 were significant for both variables in the overall population (beta $=0.002, \mathrm{p}<$ 
0.001 for apolipoprotein $\mathrm{B}$; beta $=0.002, \mathrm{p}<0.001$ for triglycerides, Table 2, Model 1). Subsequent additional adjustment for family state, i.e. spouse, NL relative or FCHL patient, did not substantially affect these associations (Table 2, Model 2). However, the overall model goodness of fit increased significantly for both apolipoprotein B and triglycerides when family state was added to the model $(\mathrm{p}<0.001$ for the $\mathrm{R}$ squared change for both variables, Table 2). This was explained by the variable FCHL patient (yes/no) in case of apolipoprotein B $(p<0.001)$ and by the variables FCHL patient (yes/no) and NL relative (yes/no) in case of triglycerides $(\mathrm{p}<0.001$ and $\mathrm{p}=0.04$, respectively). These results indicate that other factors besides PCSK9 also contribute to the FCHL phenotype.

Of interest, additional adjustment for HOMA-IR did not affect the model for apolipoprotein B (Model 3, Table 2), whereas the overall model goodness of fit for plasma triglycerides changed from 0.50 to 0.63 ( $p<0.001$, Model 3 , Table 2). PCSK9 remained a significant determinant of both FCHL diagnostic hallmarks.

Associations of plasma PCSK9 levels with apolipoprotein B in lipid subfractions Since plasma PCSK9 levels were significantly associated with both plasma triglycerides (which are predominantly present in VLDL particles) and apolipoprotein B levels (which represent particularly LDL particle number, but also IDL and VLDL particle number), we subsequently measured apolipoprotein B concentrations in VLDL and LDL subfractions in a sample of FCHL patients and NL relatives $(n=59)$, as previously reported [21]. Of note, this subset of subjects displayed similar age, sex distribution and apolipoprotein $\mathrm{B}$ levels when compared to the FCHL patients and NL relatives presented in Table 1 (data not shown). Figure 2 shows that plasma PCSK9 levels were not significantly related with VLDL apolipoprotein B levels (= VLDL particle number) $(\mathrm{r}=0.09, \mathrm{p}=0.49$, Figure 2, panel A), whereas a significant relation was observed for LDL apolipoprotein B levels ( $\mathrm{r}$ $=0.31, \mathrm{p}=0.02$, Figure 2, panel B).

\section{Heritability of plasma PCSK9 levels}

Since plasma PCSK9 levels were significantly associated with the FCHL phenotype, we subsequently estimated to what extent plasma PCSK9 levels could be accounted for by genetic factors. Heritability calculations were conducted in all FCHL relatives, i.e. the NL relatives and FCHL patients combined, resulting in 188 sibling pairs in total. The intraclass correlation (r) estimated with SAGE FCOR [19] in these 188 sibling pairs was $0.42 \pm 0.05(\mathrm{p}<0.0001)$, corresponding to a maximum heritability of $0.84\left(2 \mathrm{r}=\mathrm{h}^{2}\right)$. Sexspecified analyses also yielded significant outcomes: brother-brother (59 sibling pairs): $\mathrm{r}$ $=0.22 \pm 0.11, \mathrm{p}=0.05$; sister-brother (84 sibling pairs): $0.33 \pm 0.04, \mathrm{p}<0.0001$; sistersister ( 45 sibling pairs): $0.44 \pm 0.08, \mathrm{p}<0.0001$. These maximum heritability estimates were high when compared to the estimates for apolipoprotein $\mathrm{B}$ and triglycerides: $\mathrm{r}=$ $0.05 \pm 0.05, p=0.30$ and $r=0.16 \pm 0.05, p=0.0006$, respectively.

Heritability estimates for PCSK9 levels when all family relationships were included, as estimated with SOLAR [20], were maximally $0.67 \pm 0.19(\mathrm{p}<0.0001)$. The heritability of apolipoprotein B and triglycerides were $0.25 \pm 0.21(\mathrm{p}=0.10)$ and $0.67 \pm 0.22(\mathrm{p}<$ $0.0001)$, respectively. 
Plasma PCSK9 levels before and after treatment with atorvastatin

Previous studies have demonstrated that plasma PCSK9 levels rise with statin therapy $[22,23]$. To study whether a similar effect can be observed in a population characterized by elevated plasma PCSK9 levels, we measured PCSK9 levels in eleven FCHL patients treated with atorvastatin $40 \mathrm{mg}$ once daily for a period of eight weeks. LDL apolipoprotein B levels (interquartile range) decreased from $1.2 \mathrm{~g} / \mathrm{L}(0.9-1.3)$ to $0.7 \mathrm{~g} / \mathrm{L}$ $(0.6-0.8)(\mathrm{p}<0.001)$. Median plasma PCSK9 increased significantly from $122.0 \mathrm{ng} / \mathrm{mL}$ $(81.9-188.0 \mathrm{ng} / \mathrm{mL})$ to $150.0 \mathrm{ng} / \mathrm{mL}(113.0-228.0 \mathrm{ng} / \mathrm{mL})$ after eight weeks of treatment $(\mathrm{p}=0.018$, Figure 2$)$. The change in LDL apolipoprotein B levels was not associated with the change in PCSK9 levels in this small cohort $(r=-0.18, p=0.6)$.

\section{Discussion}

The present study demonstrated that elevated circulating PCSK9 levels are a consistent feature of FCHL. Heritability calculations showed that a substantial proportion of the variation in plasma PCSK9 levels is accounted for by genetic factors. Our results therefore suggest that plasma PCSK9 levels are involved in the complex genetic background of FCHL.

Further analyses revealed that plasma PCSK9 levels are related to both diagnostic hallmarks of FCHL, i.e. plasma triglycerides and apolipoprotein B. Nevertheless, FCHL status remained associated with both hallmarks independent of PCSK9, implying that PCSK9 is not solely responsible for the FCHL phenotype. Indeed, previous reports and also the current study have attributed an important role to insulin resistance, as reflected by HOMA-IR, in the development of the hypertriglyceridemic phenotype [24]. Furthermore, several other genetic defects have already been implicated in FCHL, such as upstream transcription factor 1 (USF1) and the apolipoprotein A1/C3/A4/A5 gene cluster $[3,4]$.

The relation between plasma PCSK 9 and apolipoprotein B levels, reflecting the total number of atherogenic particles in plasma, was largely explained by LDL particle number, as apolipoprotein B levels measured in the LDL - but not in the VLDL subfraction were also related with PCSK9. These results are in concordance with the recently published association between LDL particle clearance and plasma PCSK9 levels, i.e. PCSK9 is negatively associated with LDL particle clearance and thus positively related with LDL particle number in plasma [25]. The explanation for the relation of circulating PCSK9 with plasma triglycerides levels in FCHL is, however, less clear. Although recent studies have linked PCSK9 to VLDL production [7, 8], we did not observe a relation between VLDL apolipoprotein B and PCSK9. However, the current study was not able to distinguish between VLDL production and clearance, which together determine VLDL apolipoprotein B levels.

Several explanations could be put forward for the high circulating PCSK9 levels in FCHL. A genetic cause is very likely, given the high heritability estimates that were observed for plasma PCSK9 (ranging from 67 to 84\%). This novel finding implies that a maximum of $67-84 \%$ of the variability in plasma PCSK9 levels are accounted for by 
genetic factors. It should however be noted that also other factors, such as a shared environment, can contribute to these heritability estimates. Nevertheless, these values were higher than the estimates for the FCHL diagnostic hallmarks $(10-25 \%$ for apolipoprotein B and 32-67\% for triglycerides), which are in line with our previous report [9]. These outcomes advocate the use of the PCSK9 trait in the genetic delineation of complex lipid disorders, such as FCHL.

A direct, genetic explanation for the high PCSK9 levels in FCHL could be mutations in the PCSK9 gene itself. Some of these mutations have been associated with altered plasma PCSK9 levels [26]. Of interest, the PCSK9 gene is located on chromosome 1p32, which is nearby a locus that has repeatedly been associated with plasma apolipoprotein B levels in quantitative trait locus (QTL) linkage analyses [27-29]. Furthermore, Abifadel et al. have demonstrated that mutations in the PCSK9 gene may be responsible for hyperlipidemia in some, but not all FCHL pedigrees [30].

An alternative, indirect explanation for the elevated plasma PCSK9 levels could be found in the pathways that determine the expression of PCSK9, either genetic or environmental in origin. PCSK9 is under transcriptional control of at least two proteins, i.e. sterol regulatory element binding protein 2 (SREBP2) and hepatocyte nuclear factor 1 alpha [31-33]. Of interest, SREBP2 has also other downstream effects, such as increased LDL receptor expression and increased cholesterol synthesis [6,34]. In this light it is of interest that van Himbergen et al recently reported that plasma lathosterol levels, a plasma marker of cholesterol synthesis, are consistently elevated in FCHL patients [35]. Elevated plasma PCSK9 and lathosterol levels in FCHL patients could therefore point to a common causative pathway, i.e. SREBP 2 activation, a possibility that deserves further investigation. Of note, a positive relation between plasma lathosterol and PCSK9 levels has recently been demonstrated in cohorts of subjects after prolonged fasting [36, 37].

SREBP2 activation also accounts for the statin-induced expression of the LDL receptor and the subsequent reduction in LDL cholesterol levels [6]. Previous reports have demonstrated that statin therapy is accompanied by a rise in plasma PCSK9 levels [22, 23], which leads to the degradation of the LDL receptor and is thought to blunt the cholesterol lowering effects of statin therapy [6]. It has recently been speculated that the physiological SREBP2 mediated suppression of PCSK9 - as observed during prolonged fasting - aims to preserve plasma LDL cholesterol levels [16]. Apparently, nonphysiological impairment of HMG-CoA reductase and subsequent stimulation of PCSK9 and the LDL receptor results in a net decrease of LDL cholesterol levels. In this respect, the observed increase in PCSK9 levels after statin therapy in FCHL patients is not unique, although previous reports have not confined their findings specifically to this entity $[22,23]$. It is nevertheless of clinical relevance to report this observation since it suggests that in particular FCHL patients, who are characterized by already elevated PCSK9 levels, could benefit from PCSK9 antagonizing therapy to augment the effects of statin therapy. These antagonizing drugs are currently under development [38].

In conclusion, the present study has demonstrated that plasma PCSK9 is a highly heritable trait that is associated with both diagnostic hallmarks of FCHL. Our results 
warrant further genetic studies with plasma PCSK9 as a trait (potentially combined with lathosterol levels) to gain more insight in the development of the FCHL phenotype. Furthermore, the further rise in plasma PCSK9 levels after initiation of statin therapy suggests that FCHL patients in particular could benefit from PCSK9 antagonizing therapy.

Acknowledgements: Jason Troutt, Angela Bonner Freeman and Robert Konrad are employed by Eli Lilly and Company and own stock in Eli Lilly and Company

\section{References}

1. Goldstein, J.L., Schrott, H.G., Hazzard, W.R., Bierman, E.L., Motulsky, A.G. (1973) Hyperlipidemia in coronary heart disease. II. Genetic analysis of lipid levels in 176 families and delineation of a new inherited disorder, combined hyperlipidemia. J Clin Invest. 52, 1544-1568

2. Brouwers, M.C., de Graaf, J., van Greevenbroek, M.M., Schaper, N., Stehouwer, C.D., Stalenhoef, A.F. (2010) Novel drugs in familial combined hyperlipidemia: lessons from type 2 diabetes mellitus. Curr Opin Lipidol. 21, 530-538

3. Pajukanta, P., Lilja, H.E., Sinsheimer, J.S., Cantor, R.M., Lusis, A.J., Gentile, M., Duan, X.J., Soro-Paavonen, A., Naukkarinen, J., Saarela, J., Laakso, M., Ehnholm, C., Taskinen, M.R., Peltonen, L. (2004) Familial combined hyperlipidemia is associated with upstream transcription factor 1 (USF1). Nat Genet. 36, 371-376

4. Wojciechowski, A.P., Farrall, M., Cullen, P., Wilson, T.M., Bayliss, J.D., Farren, B., Griffin, B.A., Caslake, M.J., Packard, C.J., Shepherd, J., et al. (1991) Familial combined hyperlipidaemia linked to the apolipoprotein AI-CII-AIV gene cluster on chromosome 11q23-q24. Nature. 349, 161-164

5. Hoffer, M.J., Bredie, S.J., Boomsma, D.I., Reymer, P.W., Kastelein, J.J., de Knijff, P., Demacker, P.N., Stalenhoef, A.F., Havekes, L.M., Frants, R.R. (1996) The lipoprotein lipase (Asn291-->Ser) mutation is associated with elevated lipid levels in families with familial combined hyperlipidaemia. Atherosclerosis. 119, 159-167

6. Lambert, G. (2007) Unravelling the functional significance of PCSK9. Curr Opin Lipidol. 18, 304-309

7. Herbert, B., Patel, D., Waddington, S.N., Eden, E.R., McAleenan, A., Sun, X.M., Soutar, A.K. (2010) Increased secretion of lipoproteins in transgenic mice expressing human D374Y PCSK9 under physiological genetic control. Arterioscler Thromb Vasc Biol. 30, 1333-1339

8. Ouguerram, K., Chetiveaux, M., Zair, Y., Costet, P., Abifadel, M., Varret, M., Boileau, C., Magot, T., Krempf, M. (2004) Apolipoprotein B100 metabolism in autosomal-dominant hypercholesterolemia related to mutations in PCSK9. Arterioscler Thromb Vasc Biol. 24, 1448-1453

9. Brouwers, M.C., Cantor, R.M., Kono, N., Yoon, J.L., van der Kallen, C.J., Bilderbeek-Beckers, M.A., van Greevenbroek, M.M., Lusis, A.J., de Bruin, T.W. (2006) Heritability and genetic loci of fatty liver in familial combined hyperlipidemia. J Lipid Res. 47, 2799-2807

10. Sniderman, A.D., Castro Cabezas, M., Ribalta, J., Carmena, R., de Bruin, T.W., de Graaf, J., Erkelens, D.W., Humphries, S.E., Masana, L., Real, J.T., Talmud, P.J., 
Taskinen, M.R. (2002) A proposal to redefine familial combined hyperlipidaemia -- third workshop on FCHL held in Barcelona from 3 to 5 May 2001, during the scientific sessions of the European Society for Clinical Investigation. Eur J Clin Invest. 32, 71-73 11. Brouwers, M.C., Bilderbeek-Beckers, M.A., Georgieva, A.M., van der Kallen, C.J., van Greevenbroek, M.M., de Bruin, T.W. (2007) Fatty liver is an integral feature of familial combined hyperlipidaemia: relationship with fat distribution and plasma lipids. Clin Sci (Lond). 112, 123-130

12. Brouwers, M.C., Reesink, K.D., van Greevenbroek, M.M., Meinders, J.M., van der Kallen, C.J., Schaper, N., Hoeks, A.P., Stehouwer, C.D. (2009) Increased arterial stiffness in familial combined hyperlipidemia. J Hypertens. 27, 1009-1016

13. Matthews, D.R., Hosker, J.P., Rudenski, A.S., Naylor, B.A., Treacher, D.F., Turner, R.C. (1985) Homeostasis model assessment: insulin resistance and beta-cell function from fasting plasma glucose and insulin concentrations in man. Diabetologia. 28, 412-419

14. Welder, G., Zineh, I., Pacanowski, M.A., Troutt, J.S., Cao, G., Konrad, R.J. (2010) High-dose atorvastatin causes a rapid sustained increase in human serum PCSK9 and disrupts its correlation with LDL cholesterol. J Lipid Res. 51, 2714-2721

15. Alborn, W.E., Cao, G., Careskey, H.E., Qian, Y.W., Subramaniam, D.R., Davies, J., Conner, E.M., Konrad, R.J. (2007) Serum proprotein convertase subtilisin kexin type 9 is correlated directly with serum LDL cholesterol. Clin Chem. 53, 1814-1819

16. Persson, L., Cao, G., Stahle, L., Sjoberg, B.G., Troutt, J.S., Konrad, R.J., Galman, C., Wallen, H., Eriksson, M., Hafstrom, I., Lind, S., Dahlin, M., Amark, P., Angelin, B., Rudling, M. (2010) Circulating proprotein convertase subtilisin kexin type 9 has a diurnal rhythm synchronous with cholesterol synthesis and is reduced by fasting in humans. Arterioscler Thromb Vasc Biol. 30, 2666-2672

17. Redgrave, T.G., Roberts, D.C., West, C.E. (1975) Separation of plasma lipoproteins by density-gradient ultracentrifugation. Anal Biochem. 65, 42-49

18. Karpe, F., Hamsten, A. (1994) Determination of apolipoproteins B-48 and B-100 in triglyceride-rich lipoproteins by analytical SDS-PAGE. J Lipid Res. 35, 1311-1317 19. Sorant, A.J.M., Bonney, G.E., Elstron, R.C., Bailey-Wilson, J.E., Wilson, A.F. (1994) S.A.G.E.: Statistical Analysis for Genetic Epidemiology, Case Western Reserve University, Cleveland, Oh

20. Almasy, L., Blangero, J. (1998) Multipoint quantitative-trait linkage analysis in general pedigrees. Am J Hum Genet. 62, 1198-1211

21. Brouwers, M.C., van Greevenbroek, M.M., Bilderbeek-Beckers, M.A., RobertusTeunissen, M.G., van der Kallen, C.J., Stehouwer, C.D., de Bruin, T.W. (2007) Fatty liver--based identification of two distinct hypertriglyceridemic subgroups in familial combined hyperlipidemia. Metabolism. 56, 1311-1317

22. Careskey, H.E., Davis, R.A., Alborn, W.E., Troutt, J.S., Cao, G., Konrad, R.J. (2008) Atorvastatin increases human serum levels of proprotein convertase subtilisin/kexin type 9. J Lipid Res. 49, 394-398

23. Costet, P., Hoffmann, M.M., Cariou, B., Guyomarc'h Delasalle, B., Konrad, T., Winkler, K. (2010) Plasma PCSK9 is increased by fenofibrate and atorvastatin in a nonadditive fashion in diabetic patients. Atherosclerosis. 212, 246-251

24. Veerkamp, M.J., de Graaf, J., Stalenhoef, A.F. (2005) Role of insulin resistance in familial combined hyperlipidemia. Arterioscler Thromb Vasc Biol. 25, 1026-1031 
25. Chan, D.C., Lambert, G., Barrett, P.H., Rye, K.A., Ooi, E.M., Watts, G.F. (2009) Plasma proprotein convertase subtilisin/kexin type 9: a marker of LDL apolipoprotein B100 catabolism? Clin Chem. 55, 2049-2052

26. Humphries, S.E., Neely, R.D., Whittall, R.A., Troutt, J.S., Konrad, R.J., Scartezini, M., Li, K.W., Cooper, J.A., Acharya, J., Neil, A. (2009) Healthy individuals carrying the PCSK9 p.R46L variant and familial hypercholesterolemia patients carrying PCSK9 p.D374Y exhibit lower plasma concentrations of PCSK9. Clin Chem. 55, 21532161

27. Cantor, R.M., de Bruin, T., Kono, N., Napier, S., van Nas, A., Allayee, H., Lusis, A.J. (2004) Quantitative trait loci for apolipoprotein B, cholesterol, and triglycerides in familial combined hyperlipidemia pedigrees. Arterioscler Thromb Vasc Biol. 24, 19351941

28. Brouwers, M.C., Kono, N., van Greevenbroek, M.M., van der Kallen, C.J., Lusis, A.J., de Bruin, T.W., Cantor, R.M. (2006) Longitudinal differences in familial combined hyperlipidemia quantitative trait loci. Arterioscler Thromb Vasc Biol. 26, e118-119 29. Allayee, H., Krass, K.L., Pajukanta, P., Cantor, R.M., van der Kallen, C.J., Mar, R., Rotter, J.I., de Bruin, T.W., Peltonen, L., Lusis, A.J. (2002) Locus for elevated apolipoprotein B levels on chromosome 1p31 in families with familial combined hyperlipidemia. Circ Res. 90, 926-931

30. Abifadel, M., Bernier, L., Dubuc, G., Nuel, G., Rabes, J.P., Bonneau, J., Marques, A., Marduel, M., Devillers, M., Munnich, A., Erlich, D., Varret, M., Roy, M., Davignon, J., Boileau, C. (2008) A PCSK9 variant and familial combined hyperlipidaemia. J Med Genet. 45, 780-786

31. Dong, B., Wu, M., Li, H., Kraemer, F.B., Adeli, K., Seidah, N.G., Park, S.W., Liu, J. (2010) Strong induction of PCSK9 gene expression through HNF1 alpha and SREBP2: mechanism for the resistance to LDL-cholesterol lowering effect of statins in dyslipidemic hamsters. J Lipid Res. 51, 1486-1495

32. Jeong, H.J., Lee, H.S., Kim, K.S., Kim, Y.K., Yoon, D., Park, S.W. (2008) Steroldependent regulation of proprotein convertase subtilisin/kexin type 9 expression by sterol-regulatory element bin ding protein-2. J Lipid Res. 49, 399-409

33. Li, H., Dong, B., Park, S.W.. Lee, H.S., Chen, W., Liu, J. (2009) Hepatocyte nuclear factor 1 alpha plays a critical role in PCSK9 gene transcription and regulation by the natural hypocholesterolemic compound berberine. J Biol Chem. 284, 28885-28895

34. Horton, J.D., Goldstein, J.L., Brown, M.S. (2002) SREBPs: activators of the complete program of cholesterol and fatty acid synthesis in the liver. J Clin Invest. 109, $1125-1131$

35. van Himbergen, T.M., Otokozawa, S., Matthan, N.R., Schaefer, E.J., Buchsbaum, A., Ai, M., van Tits, L.J., de Graaf, J., Stalenhoef, A.F. (2010) Familial combined hyperlipidemia is associated with alterations in the cholesterol synthesis pathway. Arterioscler Thromb Vasc Biol. 30, 113-120

36. Browning, J.D., Horton, J.D. (2010) Fasting reduces plasma proprotein convertase, subtilisin/kexin type 9 and cholesterol biosynthesis in humans. J Lipid Res. 51,3359-3363

37. Persson, L., Cao, G., Stahle, L., Sjoberg, B., Troutt, J.S., Konrad, R.J., Galman, C., Wallen, H., Eriksson, M., Hafstrom, I., Lind, S., Dahlin, M., Amark, P., Angelin, B., Rudling, M. (2010) Circulating Proprotein Convertase Subtilisin Kexin Type 9 Has a 
Diurnal Rhythm Synchronous With Cholesterol Synthesis and Is Reduced by Fasting in Humans. Arterioscler Thromb Vasc Biol.

38. Chan, J.C., Piper, D.E., Cao, Q., Liu, D., King, C., Wang, W., Tang, J., Liu, Q., Higbee, J., Xia, Z., Di, Y., Shetterly, S., Arimura, Z., Salomonis, H., Romanow, W.G., Thibault, S.T., Zhang, R., Cao, P., Yang, X.P., Yu, T., Lu, M., Retter, M.W., Kwon, G., Henne, K., Pan, O., Tsai, M.M., Fuchslocher, B., Yang, E., Zhou, L., Lee, K.J., Daris, M., Sheng, J., Wang, Y., Shen, W.D., Yeh, W.C., Emery, M., Walker, N.P., Shan, B., Schwarz, M., Jackson, S.M. (2009) A proprotein convertase subtilisin/kexin type 9 neutralizing antibody reduces serum cholesterol in mice and nonhuman primates. Proc Natl Acad Sci U S A. 106, 9820-9825 


\section{Figure legends}

Figure 1 Association between plasma PCSK9 levels and apolipoprotein B concentrations in VLDL and LDL subfractions (measured by ultracentrifugation) in FCHL patients (closed circles) and normolipidemic relatives (open circles)

Figure 2 Plasma PCSK9 levels before and after treatment with atorvastatin $40 \mathrm{mg}$ in FCHL patients $(\mathrm{n}=11)$. Analyzed with paired-samples T-test 
Table 1. Characteristics of FCHL patients, normolipidemic (NL) relatives and their spouses

Spouses $\quad$ NL relatives $\quad$ FCHL patients

Male / Female, $\mathrm{n}$

$39 / 33$

$65 / 74$

$17 / 28$

Age, years

$51 \pm 12$

$45 \pm 15^{*}$

$54 \pm 13$

Current smoking, \%

25

26

27

BMI, kg/m²

$25.4 \pm 4.1$

$25.2 \pm 4.4$

$28.0 \pm 3.9^{\dagger}$

Waist circumference, $\mathrm{cm}$

$91.1 \pm 12.7$

$92.5 \pm 12.4^{\dagger}$

$99.2 \pm 10.4^{\dagger}$

Systolic blood pressure, $\mathrm{mmHg}$

$131 \pm 19$

$132 \pm 18^{\dagger}$

$142 \pm 17^{\dagger}$

Diastolic blood pressure, $\mathrm{mmHg}$

$83 \pm 11$

$84 \pm 10$

$88 \pm 10^{\dagger}$

Antihypertensive medication, $\%$

14

$20^{\dagger}$

$42^{\dagger}$

Triglycerides, mmol/L

$1.1(0.8-1.7)$

$1.2(0.9-1.7)^{\dagger}$

$2.0(1.8-3.3)^{\dagger}$

Apolipoprotein B, g/L

$1.0 \pm 0.2$

$1.0 \pm 0.3$

$1.4 \pm 0.2^{\dagger}$

Total cholesterol, $\mathrm{mmol} / \mathrm{L}$

$5.4 \pm 1.0$

$5.5 \pm 1.8$

$7.0 \pm 1.1^{\dagger}$

HDL cholesterol, mmol/L

$1.0 \pm 0.2$

$1.0 \pm 0.3$

$0.9 \pm 0.3^{\dagger}$

Lipid lowering medication, $\%$

8

$21^{\dagger}$

$52^{\dagger}$

HOMA-IR

$0.7(0.3-1.2)$

$0.9(0.3-1.3)^{\dagger}$

$1.3(0.9-1.8)^{\dagger}$

$* \mathrm{p}<0.05$ versus spouses

$\uparrow \mathrm{p}<0.05$ versus spouses, age- and sex-adjusted 
Table 2. Associations of plasma PCSK9 with apolipoprotein B and triglycerides levels

\begin{tabular}{l|cccc|cccc}
\hline & \multicolumn{4}{|c|}{ Apolipoprotein B } & \multicolumn{3}{c}{ Triglycerides } \\
\cline { 2 - 8 } & $\begin{array}{c}\text { Beta } \\
\text { (PCSK9) }\end{array}$ & $\begin{array}{c}\mathrm{p} \text {-value } \\
\text { (PCSK9) }\end{array}$ & $\begin{array}{c}\mathrm{R} \text { for } \\
\text { model }\end{array}$ & $\begin{array}{c}\text { p-value for } \\
\text { R squared change* }\end{array}$ & $\begin{array}{c}\text { Beta } \\
\text { (PCSK9) }\end{array}$ & $\begin{array}{c}\mathrm{p} \text {-value } \\
\text { (PCSK9) }\end{array}$ & $\begin{array}{c}\text { R for } \\
\text { model }\end{array}$ & $\begin{array}{c}\text { p-value for } \\
\text { R squared change* }\end{array}$ \\
\hline Model 1 & 0.002 & $<0.001$ & 0.37 & & 0.002 & $<0.001$ & 0.38 \\
Model 2 & 0.001 & 0.007 & 0.59 & $<0.001$ & 0.001 & 0.003 & 0.50 & $<0.001$ \\
\hline
\end{tabular}

Unstandardized beta's (with p-value) represent regression slopes for PCSK9

Model 1: adjustment for age and sex

Model 2: adjustment for age, sex and family state (i.e. NL relative [yes/no] and FCHL patient [yes/no])

Model 3: adjustment for age, sex, family state (i.e. NL relative [yes/no] and FCHL patient [yes/no]) and HOMA-IR

$*$ p-values represent the $\mathrm{R}$ squared change when compared to the previous model 


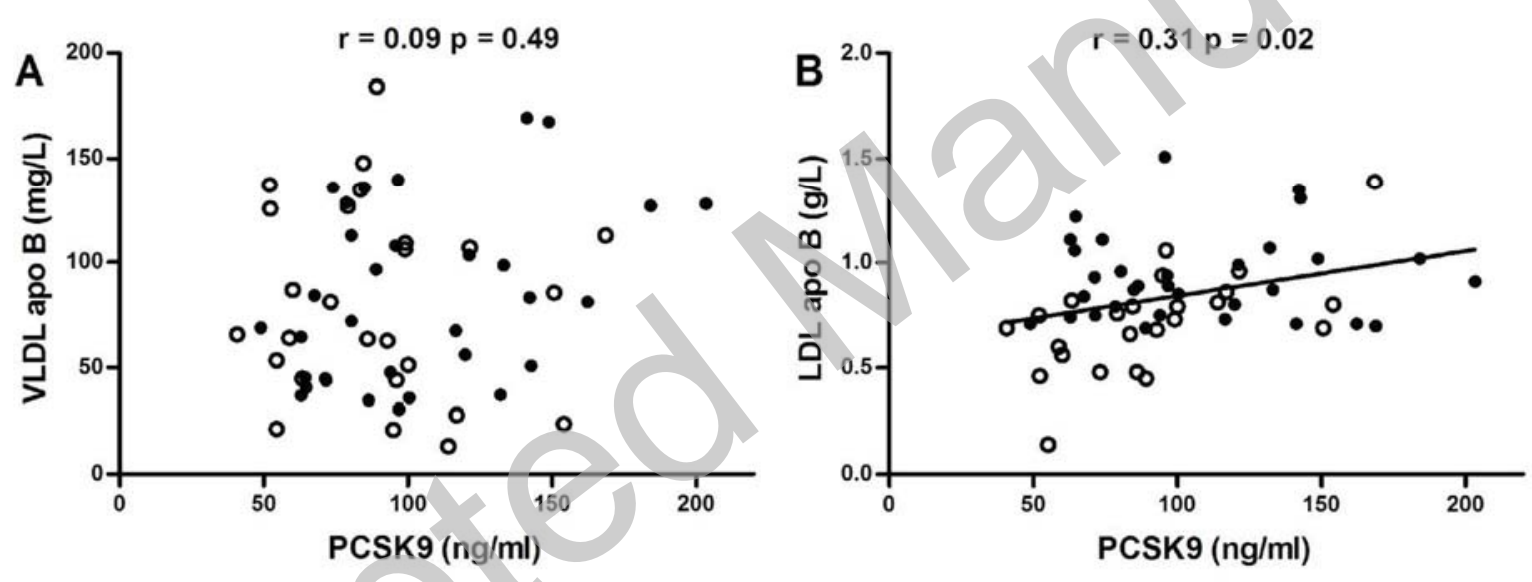




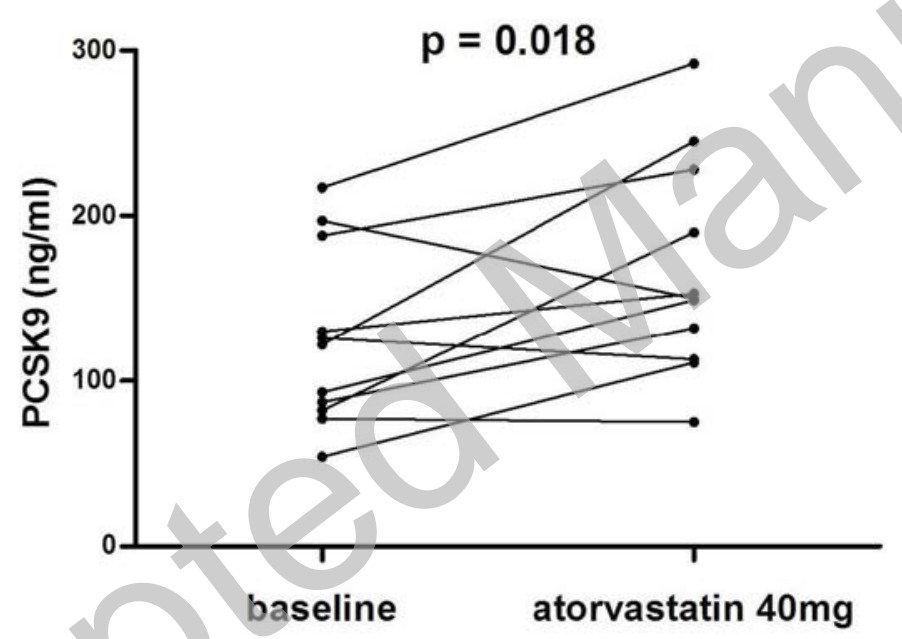

Licenced copy. Copying is not permitted, except with prior permission and as allowed by law. (C) 2011 The Authors Journal compilation (C 2011 Portland Press Limited 